\title{
Preventive effect of chrysin on experimental autoimmune uveitis triggered by injection of human IRBP peptide 1-20 in mice
}

\begin{abstract}
Xiangda Meng, Sijie Fang, Zhuhong Zhang, Yang Wang, Caiyun You, Jingkai Zhang and Hua Yan
Uveitis is a common cause of blindness worldwide. Experimental autoimmune uveitis (EAU) is an animal model of noninfectious uveitis. Chrysin (5,7-dihydroxyflavone) is a member of the flavonoid family and has anti-inflammatory effects. We immunized C57BL/6J mice with human interphotoreceptor retinoid-binding protein peptide 1-20 to induce EAU. Chrysin was administered intragastrically at $25 \mathrm{mg} / \mathrm{kg}$ daily to the chrysin-treated mice from 3 days before immunization to 21 days after immunization. Vehicle was administered to the mice in the control group according to the same protocol. Lower clinical and histopathological scores, increased integrity of the blood-retinal barrier (BRB) and higher expression of tight junction proteins were observed in the chrysin-treated mice. Chrysin significantly decreased the proportions of Th1, Th17 and $\mathrm{CD} 4{ }^{+} \mathrm{CD} 3^{+} \mathrm{CD} 62 \mathrm{~L}^{+}$Th0 cells, and increased the proportion of Treg cells. Both macrophage infiltration and the expression of inducible nitric oxide synthase in the retina were efficiently inhibited by chrysin treatment. In chrysin-treated mice, the expression of interferon- $\gamma$, interleukin (IL)-17A, IL-6, IL-1 $\beta$ and tumor necrosis factor- $\alpha$ was reduced in the retina, whereas higher levels of transforming growth factor- $\beta$ were detected. Furthermore, NF-кBp65 was downregulated after chrysin treatment. In conclusion, as an anti-inflammatory molecule, chrysin exerts a preventive effect on EAU by modulating the balance among helper T-cell subsets and suppressing ocular inflammation, thereby maintaining the integrity of the BRB. Cellular \& Molecular Immunology (2017) 14, 702-711; doi:10.1038/cmi.2015.107; published online 21 March 2016
\end{abstract}

Keywords: blood-retinal barrier; chrysin; experimental autoimmune uveitis; NF-кB; T helper-cell subsets

\section{INTRODUCTION}

Uveitis is a common cause of blindness worldwide, and it usually affects people ranging from 20 to 59 years old. ${ }^{1}$ On the basis of its different etiologies, uveitis can be classified as infectious or noninfectious. Infectious uveitis can be caused by viruses, bacteria, fungi or parasites, and it is mainly treated through elimination of the causative pathogen. However, noninfectious uveitis is a recurrent and immune-mediated disease that usually affects the eyes bilaterally. ${ }^{2}$ It is idiopathic and is usually accompanied by immunologic abnormalities or systemic diseases. ${ }^{2}$ Cycloplegics and corticosteroids (both topical and systemic) are the mainstays of treatment for noninfectious uveitis, but a growing body of research suggests the usage of immunosuppressive or biological agents such as cyclophosphamide (CTX), ${ }^{3}$ cyclosporin $\mathrm{A}^{4}$ and infliximab. ${ }^{5,6}$
Experimental autoimmune uveitis (EAU) serves as an animal model of noninfectious uveitis. EAU in mice is induced by injecting emulsified antigen subcutaneously, which destroys the body's immune tolerance. After immunization, self-reactive antigens are first presented to naive $\mathrm{T}$ cells by antigenpresenting cells (APCs). Then, these naive $\mathrm{T}$ cells are activated and transformed into $\mathrm{CD} 4^{+} \mathrm{Th} 0$ cells that further differentiate into Th1, Th17, Treg or other T-cell types. Moreover, it is generally accepted that an imbalance among these T-cell subsets has a decisive role in the pathogenesis of EAU.,8 Th1 and Th17 cells, which serve as destructive T cells, destroy the blood-retinal barrier (BRB) by secreting cytokines such as interferon (IFN)- $\gamma$ and interleukin (IL)-17. These cytokines are capable of recruiting macrophages, leading to intraocular inflammation that is dependent on the activation of multiple

Department of Ophthalmology, Tianjin Medical University General Hospital, Tianjin 300052, China

Correspondence: Professor H Yan, M.D., Ph.D, Chief Physician, Department of Ophthalmology, Tianjin Medical University General Hospital, No. 154, Anshan Road, Tianjin 300052, China.

E-mail: phuayan2000@163.com

Received: 8 March 2015; Revised: 28 November 2015; Accepted: 29 November 2015 
signaling pathways involving nuclear factor kappa B (NF-кB). $\mathrm{NF}-\mathrm{\kappa B}$ is a nuclear transcription factor that is critical for inflammation and has a major role in the pathological immune progression of $\mathrm{EAU}^{9,10}$ because it regulates many pro-inflammatory cytokines and enzymes at the transcriptional level, such as IL- $1 \beta$, IL- 6 , tumor necrosis factor- $\alpha$ (TNF- $\alpha$ ) and inducible nitric oxide (iNOS), which can activate macrophages and induce oxidative stress.

Chrysin (5,7-dihydroxyflavone) is a member of the flavonoid family that is found in honey, propolis and many other plants. ${ }^{11}$ Flavonoids have a broad spectrum of biological activities such as antitumor, immunomodulatory, anti-inflammatory, antiallergy, antioxidant and cardioprotective effects. ${ }^{12}$ Some researchers have shown that flavonoids and their derivatives exert potential benefits in animal models of anterior uveitis. ${ }^{13,14}$ However, these studies have seldom discussed the immunological mechanisms in depth. Chrysin has been described as a potential therapeutic agent for the treatment of some inflammatory diseases such as inflammatory bowel disease ${ }^{15}$ and allergic inflammatory diseases, ${ }^{16,17}$ although the mechanisms underlying its anti-inflammatory activity are still poorly understood. Recent studies have confirmed that chrysin exerts its anti-inflammatory effects partly by inhibiting the NF- $\kappa B$ signaling pathway, ${ }^{16,18,19}$ which aroused our interest in examining chrysin's potentially protective effect on EAU. Because new therapeutic strategies for the treatment of uveitis are greatly needed, this study was designed to investigate the possible preventive effect of chrysin on EAU induced by human interphotoreceptor retinoid-binding protein peptide $1-20\left(\operatorname{IRBP}_{1-20}\right)$ immunization in mice and to discuss the potential immunological mechanisms underlying these effects.

\section{MATERIALS AND METHODS}

\section{Animals}

Female C57BL/6J mice (6-8 weeks old) purchased from the Academy of Military Medical Science (Beijing, China) were housed in specific pathogen-free conditions with water and food available ad libitum. All procedures involving the mice were approved by the Laboratory Animal Care and Use Committee of Tianjin Medical University and also conformed to the Association for Research in Vision and Ophthalmology Statement for the Use of Animals in Ophthalmic and Vision Research.

\section{Induction of EAU and treatment}

Each C57BL/6J mouse was immunized subcutaneously in one rear footpad and the tail head, and rump (total of $200 \mu \mathrm{l}$ ) with an emulsion containing $300 \mu \mathrm{g}$ of human IRBP $_{1-20}$ (GPTHLFQPSLVLDMAKVLLD, Sangon, Shanghai, China) in phosphate-buffered saline (PBS) and $300 \mu \mathrm{g}$ of Mycobacterium tuberculosis H37Ra (Difco, Detroit, MI, USA) in complete Freund's adjuvant (CFA, Sigma, St Louis, MO, USA; 1:1, v/v). Simultaneously, the mice received $1 \mu \mathrm{g}$ of Bordetella pertussis toxin (List Biological Laboratories, Campbell, CA, USA) intraperitoneally. Then the mice were randomly divided into a chrysin-treated group ( $n=30$ mice) and a control group
( $n=30$ mice). For the treatment of EAU, chrysin (Acros Organics, Morris Plains, NJ, USA) dissolved in $10 \mu \mathrm{l}$ of dimethylsulfoxide (DMSO) and $140 \mu$ l of PBS was administered at $25 \mathrm{mg} / \mathrm{kg}$ per day by oral gavage to the chrysin-treated mice from 3 days before immunization (day -3$)$ to day 21 after immunization, when the mice were killed. Vehicle containing $10 \mu \mathrm{l}$ of DMSO and $140 \mu \mathrm{l}$ of PBS was administered to the control group mice. We did not observe any unnatural animal deaths during the experimental period.

\section{Clinical and histological assessment of EAU}

After the pupils were dilated with tropicamide, the fundus oculi of each mouse was examined daily with a binocular indirect ophthalmoscope with an additional $+90 \mathrm{D}$ lens from day 12 until day 21 post immunization. At day 21, eye-ground photographs were taken with an otoendoscope (27018A, Karl Storz, Tuttlingen, Germany) computer imaging system after pupillary dilation and the application of topical corneal anesthesia (oxybuprocaine hydrochloride) with a coverslip and medical sodium hyaluronate gel (Bausch \& Lomb Freda, Shandong, China) to avoid the influence of the corneal curvature. The incidence and severity of EAU were scored according to the criteria reported by Caspi and coworkers. ${ }^{20}$ Then the eyes were enucleated and fixed in 10\% formaldehyde and 5\% glacial acetic acid/PBS. Dehydrated tissues were embedded in paraffin wax and sectioned to $5-\mu \mathrm{m}$ thicknesses. The eye sections were stained with hematoxylin and eosin (H\&E) for histopathological evaluation according to Caspi's criteria. $^{20}$

\section{Flow cytometry analysis}

Single-cell suspensions were isolated from the mouse spleens on day 21 by mashing and passing the spleens through a cell strainer $(40-\mu \mathrm{m}$ nylon, BD Falcon, San Jose, CA, USA). For Treg cell staining, single-cell suspensions were stained with anti-mouse CD4 FITC (BD Biosciences, San Jose, CA, USA), anti-mouse CD25 PerCP-Cy5.5 (BD Biosciences) and antimouse Foxp3 phycoerythrin (PE) (eBioscience, San Diego, CA, USA) antibodies. For Th0 cell staining, the cells were stained with anti-mouse CD4 FITC, anti-mouse CD3 PerCP-Cy5.5 (BD Biosciences) and anti-mouse CD62L PE (BD Biosciences) antibodies. For Th1 and Th17 cell staining, the cells were restimulated with $500 \mathrm{ng} / \mathrm{ml}$ phorbol 12-myristate 13 -acetate (Sigma), $500 \mathrm{ng} / \mathrm{ml}$ ionomycin (Sigma) and $1 \mu \mathrm{g} / \mathrm{ml}$ of brefeldin A (Biosciences) for $4 \mathrm{~h}$. The cells were then collected and stained with anti-mouse IFN- $\gamma$ PE (BD Biosciences) and antimouse CD4 FITC antibodies for the Th1 cell analysis or stained with anti-mouse IL-17 PE (BD Biosciences) and anti-mouse CD4 FITC antibodies for the Th17 cell analysis. Cells were then analyzed with a BD FACSCalibur (Becton Dickinson, Mount View, CA, USA), and the acquired data were analyzed with FlowJo 7.6 (Ashland, OR, USA).

\section{Assessment of retinal vasculature and the BRB}

A total of $100 \mu \mathrm{l}$ of $2 \%(\mathrm{w} / \mathrm{v})$ Evans Blue dye (Sigma-Aldrich, St Louis, MO, USA) was injected through the tail vein. Two 
hours later, the mice were killed and their eyes were enucleated and immediately fixed in $4 \%$ paraformaldehyde for $2 \mathrm{~h}$. Then the anterior segments were removed and the retinas were dissected and washed in cold PBS. Finally, the retinas were spread on glass slides, with the vitreous side facing up, and mounted with mounting medium. Pictures were taken with a confocal scanning laser imaging system fitted with krypton-argon lasers (FV1000, Olympus, Tokyo, Japan).

\section{Immunohistochemical staining}

Eyes were removed on day 21 and fixed in 10\% formaldehyde and $5 \%$ glacial acetic acid/PBS. Sections $(5 \mu \mathrm{m})$ were prepared from paraffin-embedded tissues and incubated overnight at $4{ }^{\circ} \mathrm{C}$ with rat monoclonal antibody to $\mathrm{F} 4 / 80$ (1:200, GeneTex, Irvine, CA, USA). Then the sections were stained with biotinylated anti-rat IgG secondary antibody (1:200, Vector Laboratories, Burlingame, CA, USA) for $2 \mathrm{~h}$, followed by horseradish peroxidase streptavidin incubation for $1 \mathrm{~h}$. Specific labeling was visualized by incubation with diaminobenzidine (Zhongshan Goldenbridge Biotechnology, Beijing, China). Finally, the sections were counterstained with hematoxylin (Solarbio Science \& Technology, Beijing, China). Photographs were taken with a Leica DMI4000B (Leica Microsystems, Hesse, Germany).

\section{Immunofluorescence}

Eyes were enucleated on day 21 and immediately incubated in optimum cutting temperature compound. After being frozen at $-80^{\circ} \mathrm{C}$ for $15 \mathrm{~min}$, eyes were cut into 5 - $\mu \mathrm{m}$-thick sections, fixed in acetone and incubated with the primary antibody (rat monoclonal to F4/80, dilution 1:200, GeneTex; rabbit polyclonal to iNOS, dilution 1:200, Abcam, Cambridge, UK) overnight at $4{ }^{\circ} \mathrm{C}$. Then the sections were incubated with secondary antibodies. Finally, the sections were treated with 4',6-diamidino-2-phenylindole (DAPI) for $1 \mathrm{~min}$ at $37^{\circ} \mathrm{C}$. Pictures were taken with a Nikon Coolscope (Nikon, Dusseldorf, Germany).

\section{Real-time quantitative PCR}

Total RNA of the retina was isolated using TRIzol reagent (Invitrogen, Carlsbad, CA, USA) and reverse transcribed into cDNA with TransScript First-Strand cDNA Synthesis SuperMix (TransGen Biotech, Beijing, China). The relative amounts of Ifng, Il17a, Il1b, Il6, Tnf, Il10, Tgfbl, Rela and Nos2 mRNA were detected by real-time quantitative PCR (qRT-PCR) with TransScript Top Green qPCR SuperMix (TransGen Biotech). Actb mRNA was used as an internal control. All procedures were performed according to the manufacturer's instructions. The primers used are shown in Table 1. The relative mRNA expression was determined with the $2^{-\Delta \Delta \mathrm{Ct}}$ method. ${ }^{21}$

\section{Western blotting}

Western blotting was performed using standard methods. Retinas and choroids were carefully dissected from the eyes, and the protein concentration was measured using a protein assay (Bradford Protein Assay; Bio-Rad, Hercules, CA, USA).
Table 1 Primer sets for qRT-PCR

\begin{tabular}{|c|c|}
\hline Gene & Sequence $\left(5^{\prime}-3^{\prime}\right)$ \\
\hline \multirow[t]{2}{*}{$I F N-\gamma$} & CTGCTGATGGGAGGAGATGT \\
\hline & TTTGTCATTCGGGTGTAGTCA \\
\hline \multirow[t]{2}{*}{$I L-17 A$} & GGACTCTCCACCGCAATGA \\
\hline & TCAGGCTCCCTCTTCAGGAC \\
\hline \multirow[t]{2}{*}{ IL-6 } & CCAGTTGCCTTCTTGGGACT \\
\hline & GGTCTGTTGGGAGTGGTATCC \\
\hline \multirow[t]{2}{*}{$I L-1 \beta$} & CACCTCACAAGCAGAGCACA \\
\hline & GCCCATACTTTAGGAAGACACG \\
\hline \multirow[t]{2}{*}{$T N F-\alpha$} & CACCACCATCAAGGACTCAA \\
\hline & GAGACAGAGGCAACCTGACC \\
\hline \multirow[t]{2}{*}{$T G F-\beta$} & ATTCCTGGCGTTACCTTGG \\
\hline & AGCCСTGTATTCCGTCTCCT \\
\hline \multirow[t]{2}{*}{ IL-10 } & TGCACTACCAAAGCCACAAG \\
\hline & TGATCCTCATGCCAGTCAGT \\
\hline \multirow[t]{2}{*}{ iNOS } & AATCTTGGAGCGAGTTGTGG \\
\hline & GCAGCCTCTTGTCTTTGACC \\
\hline \multirow[t]{2}{*}{$\beta$-actin } & GGGTCAGTGGAGTAGACAGCA \\
\hline & TGGATGTGGTAGAGCAAAGC \\
\hline
\end{tabular}

Abbreviation: qRT-PCR, real-time quantitative PCR.

Equal amounts of protein were separated by electrophoresis on $10-12 \%$ dodecyl sulfate-polyacrylamide gel and electroblotted onto polyvinylidene fluoride membranes (Millipore, Billerica, MA, USA). The membranes were blocked in 5\% skim milk for $1 \mathrm{~h}$ and incubated with antibodies to IL-1 $\beta$ (1:2000, Abcam), IL-6 (1:1000, Abcam), IL-10 (1:500, Abcam), IL-17 A (1:1000, Abcam), IFN- $\gamma$ (1:500, GeneTex), TNF- $\alpha$ (1:1000, GeneTex), TGF- $\beta$ (1:250, Cell Signaling Technology, Boston, MA, USA), p65 (1:1000, Cell Signaling Technology), iNOS (1:500, Abcam), occludin (1:250, Invitrogen) and ZO-1 (1:200, Invitrogen) overnight at $4{ }^{\circ} \mathrm{C}$. Then the membranes were washed in $0.1 \%$ triethanolamine buffered saline solution-Tween (TBS-T) and incubated with secondary antibodies at room temperature for $1 \mathrm{~h}$. Finally, the blots were scanned with a ChemiDoc MP System (Bio-Rad), and the bands were quantified by Image J software (NIH, Bethesda, MD, USA). $\beta$-actin (1:1000, Zhongshan Goldenbridge Biotechnology) was used as an internal reference.

\section{Statistical analysis}

All data are presented as the mean \pm s.d. and were analyzed by SPSS 17.0 software (Chicago, IL, USA). The Mann-Whitney $U$-test was used to compare the differences between the control and the chrysin groups. The differences were considered to be statistically significant at $P<0.05$.

\section{RESULTS}

Chrysin ameliorates the severity of EAU both clinically and histologically

To explore the therapeutic effect of chrysin on uveitis, an IRBP/ CFA-induced EAU model was used. Mice were treated with chrysin daily from day -3 to day 21 . From day 12 until day 21 fundus examinations were performed to observe the disease 

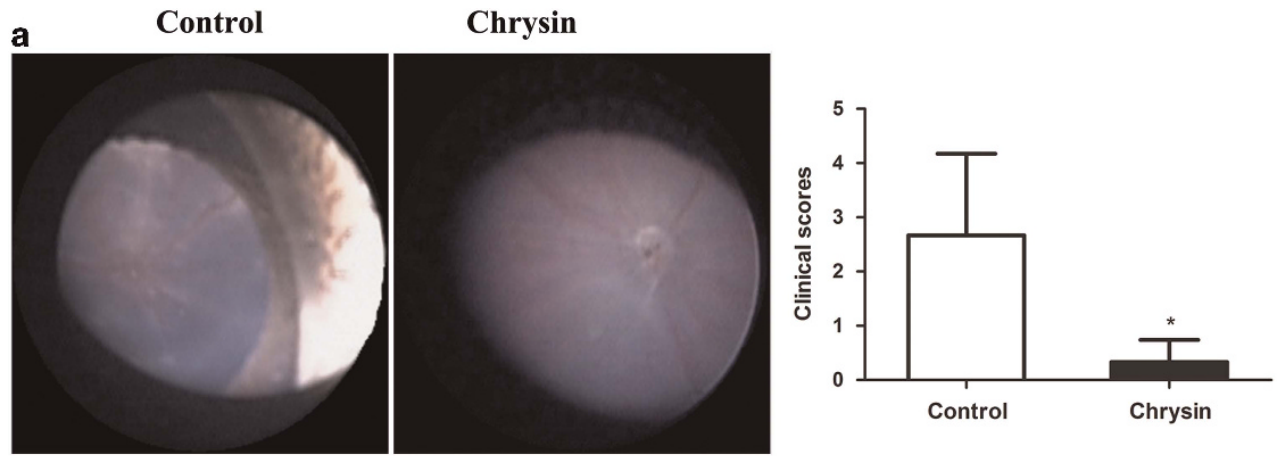

b

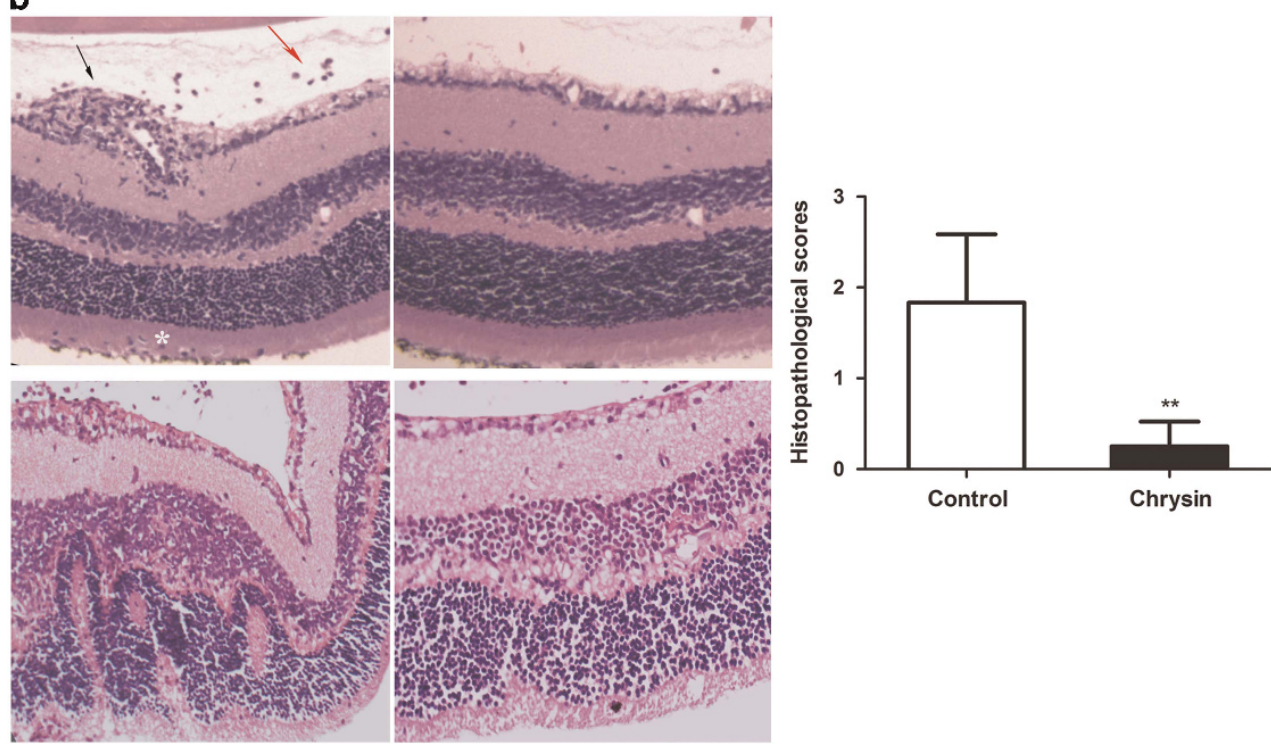

Figure 1 Effect of chrysin on the fundus and histological manifestations of EAU. (a) Fundal images of control mice and chrysin-treated mice. Retinal infiltration, vasculitis and papilledema were observed in the control mice, and these effects were scarcely found in the chrysin-treated mice. The clinical scores were significantly decreased in the chrysin-treated group compared with the control group ( ${ }^{*} P<0.01 ; n=6$ mice). (b) H\&E staining. The control mice had more severe vasculitis (black arrow), vitreous infiltration (red arrow) and photoreceptor cell layer infiltration (white asterisk), as compared with the chrysin-treated mice (original magnification $\times 100$ ). The histopathological scores were significantly decreased in the chrysin-treated group compared with the control group (**P<0.01; $n=6$ mice). EAU, experimental autoimmune uveitis; H\&E, hematoxylin and eosin.

severity and clinical course for each mouse. EAU was characterized by vasculitis, retinal infiltrations, papilledema, retinal detachment and retinal atrophy. The chrysin-treated mice showed less severe inflammation and tissue damage (Figure 1a). Clinical scoring was performed on day 21. The clinical scores $(0.33 \pm 0.41$ vs $2.67 \pm 1.51, P<0.05)$ were significantly lower in the chrysin-treated mice than in the control mice (Figure 1a).

To further validate the clinical therapeutic effects of chrysin, a histological assessment was performed on day 21 . The main histological characteristics of EAU include the infiltration of inflammatory cells, retinal folds or detachments, granulomas in the choroid and retina, and subretinal neovascularization. ${ }^{18}$ On the basis of H\&E staining, the chrysin-treated mice showed less infiltration by inflammatory cells and milder retinal folds than the control mice (Figure 1b). Histopathologic grading indicated that the chrysin-treated mice had significantly lower scores than the control mice $(0.25 \pm 0.27$ vs $1.83 \pm 0.75, P<0.01$; Figure $1 \mathrm{~b})$.

\section{Chrysin regulates the proportions of peripheral} T-lymphocyte subsets

An autoimmune ocular disease EAU is mediated by three critical populations of T cells: Th1, Th17 and Treg cells. ${ }^{22-27}$ Hence, we examined the T-lymphocyte subsets in the spleens of mice in both groups by flow cytometry. Our data demonstrated that the chrysin-treated mice, compared with control mice, had lower percentages of $\mathrm{CD}^{+} \mathrm{IFN}-\gamma^{+}$Th1 cells $(P<0.05$, Figure $2 \mathrm{a})$ and $\mathrm{CD} 4^{+} \mathrm{IL}_{1} 7^{+}$Th17 cells $(P<0.01$, Figure $2 \mathrm{~b}$ ), and a higher percentage of $\mathrm{CD} 4^{+} \mathrm{CD} 25^{+}$Treg cells $(P<0.05$, Figure $2 \mathrm{c})$ in their spleens. The percentage of $\mathrm{CD} 4^{+}$ $\mathrm{CD}^{+}{ }^{+} \mathrm{CD} 62 \mathrm{~L}^{+}$Th0 cells decreased $(P<0.01$, Figure $2 \mathrm{~d})$ in the chrysin-treated mice. 

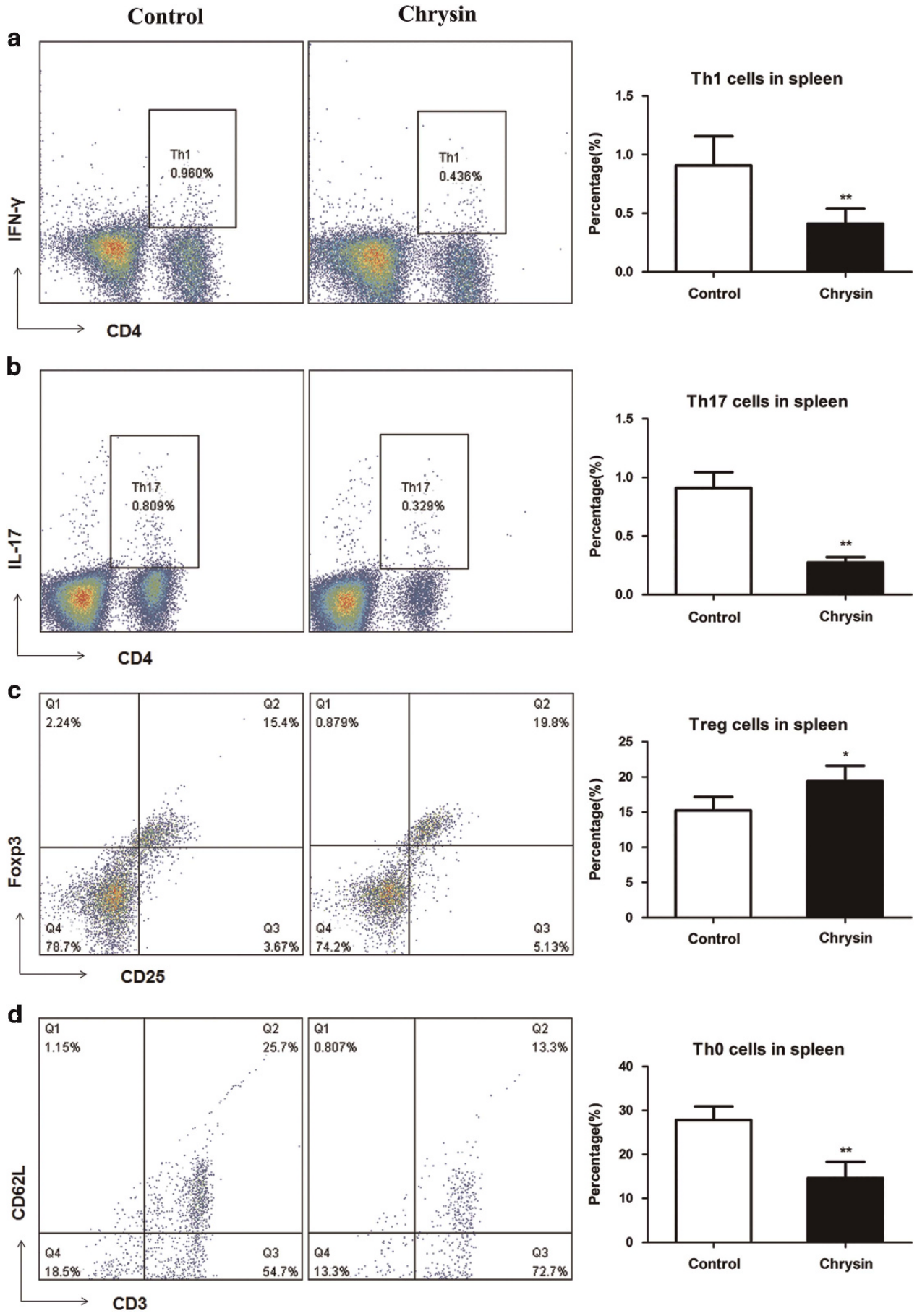

Figure 2 The effects of chrysin on the percentages of Th1, Th17, Treg and Th0 cells in the spleen. Chrysin decreased the proportion of Th1, Th17, Th0 cells but increased the proportion of Treg cells compared with that in the control mice. (a) Th1 cells, (b) Th17 cells, (c) Treg cells and (d) Th0 cells. ${ }^{*} P<0.05 ;{ }^{*} P<0.01$ ( $n=5$ mice). Data are expressed as the mean \pm s.d.

\section{Chrysin exerts a protective effect on the BRB}

Inflammation may increase the permeability of retinal and choroidal vessels, destroy the BRB and lead to cell infiltration. To evaluate whether chrysin functions in maintaining the integrity of the BRB, Evans Blue staining of the BRB and the expression of the tight junction proteins ZO-1 and occludin were measured.
Figure 3a shows the results of the Evans Blue staining in each group. A clear increase in Evans Blue leakage from the retinal vasculature was observed in the control mice compared with the chrysin-treated mice. This result was further confirmed by the expression of tight junction proteins $\mathrm{ZO}-1$ and occludin. Significant increases in the levels of ZO-1 and occludin were 
a

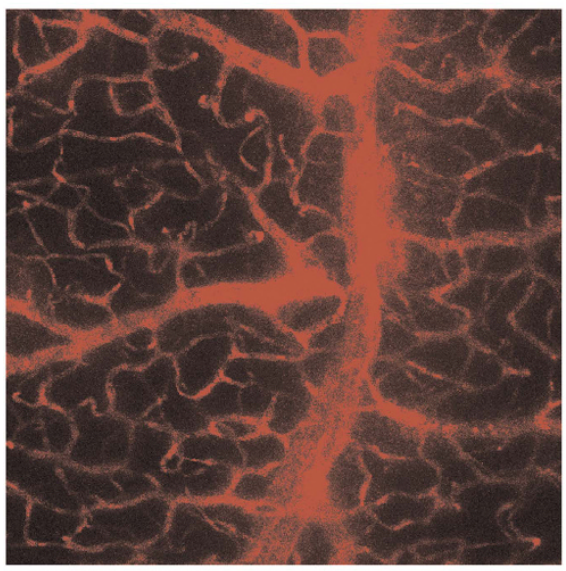

b
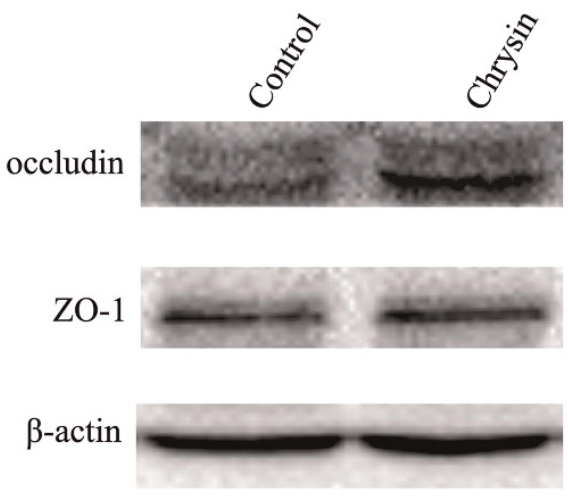

\section{Chrysin}
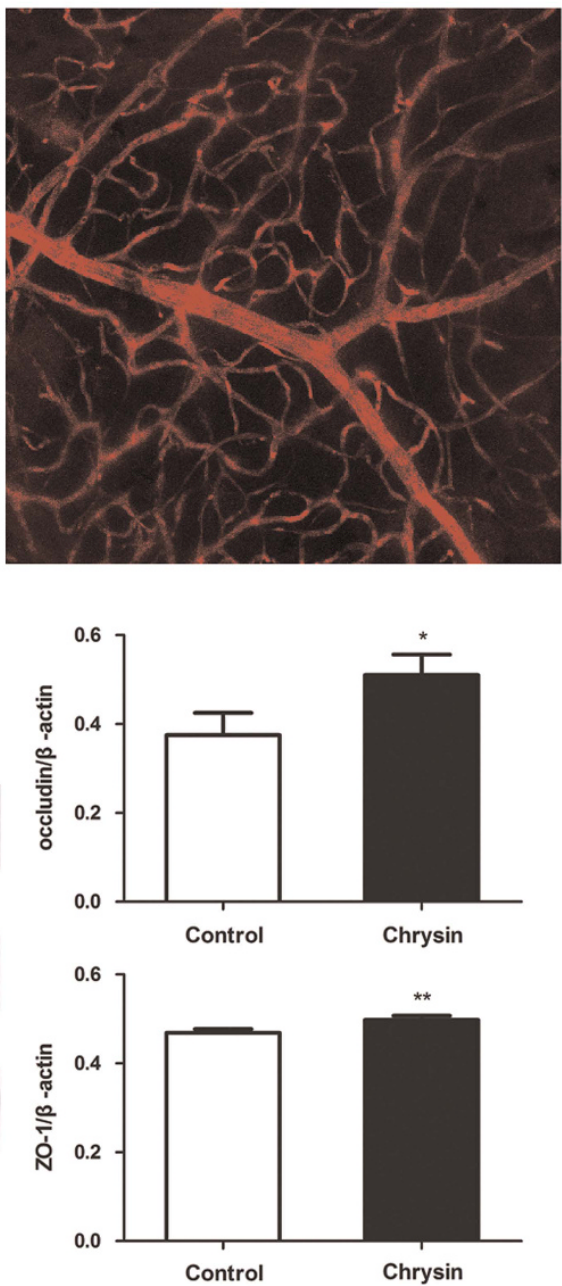

Figure 3 Chrysin's protective effect on the BRB. (a) Increased Evans Blue leakage in the control group compared with the chrysin group (original magnification $\times 200 ; n=4$ mice). (b) The levels of tight junction proteins ZO-1 and occludin were measured by western blotting. The chrysin group showed significantly increased expression of both proteins $\left({ }^{*} P<0.05 ;{ }^{*} P<0.01 ; n=6\right.$ mice). Data are expressed as the mean \pm s.d. BRB, blood-brain barrier.

observed in the chrysin-treated group compared with the control group (Figure 3b).

Chrysin blocks the infiltration of macrophages and inhibits macrophage iNOS secretion

Immunohistochemical staining was performed to detect macrophage infiltration. The chrysin-treated mice had fewer infiltrated macrophages in the vitreous and in the retina than the control mice (Figure 4a). In addition, immunofluorescence staining with anti-F4/80 and anti-iNOS antibodies showed iNOS expression in F4/80-positive macrophages. Double-positive cells were observed in the control mice but not in the chrysin-treated mice (Figure 4b).

The expression of iNOS in the retina was determined by qRT-PCR and western blotting. The relative mRNA expression of iNOS was significantly decreased $(P<0.01)$ in the chrysintreated mice compared with the control mice. In addition, a significant reduction $(P<0.01)$ in the relative protein level of iNOS was shown by western blotting (Figure 4c).

Chrysin downregulates pro-inflammatory cytokines while upregulating anti-inflammatory cytokines in the retina

To explore the effects of treatment with chrysin on intraocular cytokines at both the mRNA and protein levels, relative mRNA expression was measured by $\mathrm{qRT}-\mathrm{PCR}$, and relative protein expression was measured by western blotting. Chrysin suppressed the relative mRNA expression of pro-inflammatory cytokines, including IFN- $\gamma(P<0.05)$, IL-17A $(P<0.01)$, IL-6 $(P<0.01)$, TNF- $\alpha(P<0.01)$ and IL-1 $\beta(P<0.05)$, whereas it promoted the expression of the anti-inflammatory cytokine TGF- $\beta(P<0.05$; Figure 5$)$. The relative protein expressions of IFN- $\gamma(P<0.01)$, IL-17A $(P<0.01)$, IL-6 $(P<0.01)$, TNF- $\alpha$ $(P<0.01)$ and IL-1 $\beta(P<0.01)$ were also significantly inhibited after treatment with chrysin (Figure 6). Correspondingly, the expression of TGF- $\beta$ was upregulated in the chrysin-treated 

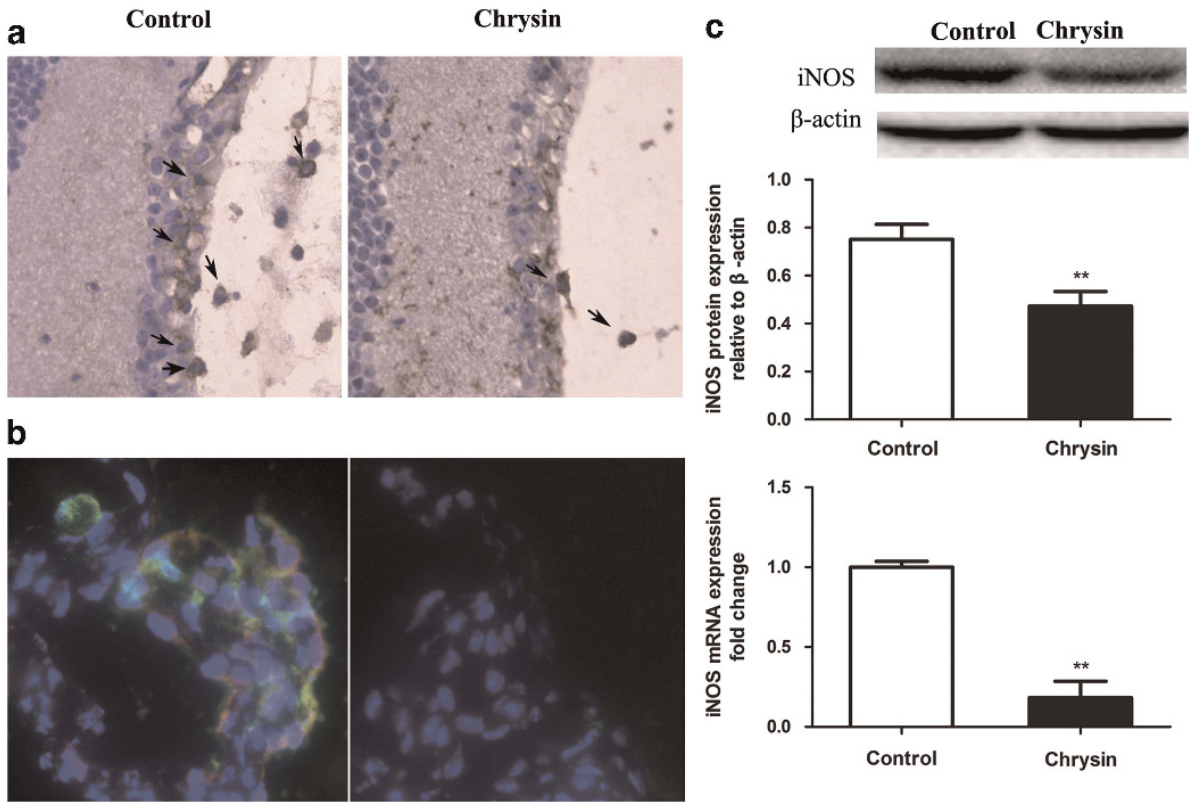

Figure 4 Macrophage infiltration and expression of iNOS. (a) IHC indicates that chrysin reduced the number of $\mathrm{F}^{2} / 80^{+}$macrophages (black arrows) that infiltrated the vitreous and the retina $\left(n=4\right.$ mice). (b) Immunofluorescence staining of ciliary processes. F4/80 ${ }^{+}$cells stain green, iNOS ${ }^{+}$cells stain red and the double-positive cells stain orange ( $n=4$ mice). (c) Chrysin suppressed the expression of iNOS. Both western blotting and qRT-PCR showed significantly decreased iNOS expression ( ${ }^{* *} P<0.01 ; n=6$ mice). Data are expressed as the mean \pm s.d. iNOS, inducible nitric oxide; qRT-PCR, real-time quantitative PCR.

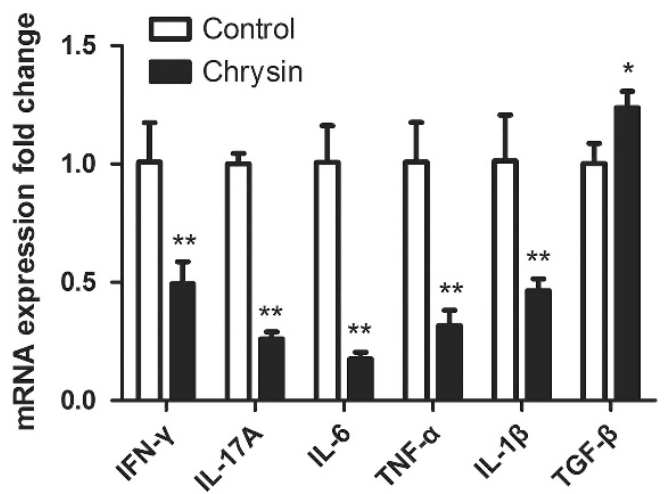

Figure 5 qRT-PCR measurement of cytokines. IFN- $\gamma$, IL-17A, IL-6, TNF- $\alpha$ and IL-1 $\beta$ mRNA showed decreased expression, whereas TGF- $\beta$ mRNA showed increased expression in the chrysin-treated mice vs the control mice $\left({ }^{*} P<0.05 ;{ }^{*} P<0.01 ; n=6\right.$ mice). Data are expressed as the mean \pm s.d. IFN- $\gamma$, interferon- $\gamma ;$ IL, interleukin; qRT-PCR, real-time quantitative PCR; TGF- $\beta$, transforming growth factor- $\beta$; TNF- $\alpha$, tumor necrosis factor- $\alpha$.

mice $(P<0.05)$. However, there was no significant change in the expression of IL-10 (data not shown) at either the mRNA or the protein level.

\section{Chrysin suppresses the expression of NF- $\mathrm{KB}$}

NF-KB participates in one of the pathways that is important in the development of EAU. ${ }^{9,10}$ NF- $\mathrm{kBp} 65$, a subunit of NF- $\mathrm{kB}$, is commonly used as a marker to evaluate NF- $\mathrm{\kappa B}$ signaling. Both the relative mRNA and protein expression levels of NF- $\mathrm{kBp} 65$ decreased significantly $(P<0.01)$ in chrysin-treated mice vs control mice (Figure 7).

\section{DISCUSSION}

Several studies that have examined ocular disease have focused on the anti-inflammatory effects of natural products such as green tea, ${ }^{28,29}$ curcumin, $^{30}$ flavonoids ${ }^{13}$ and flavonoid derivatives. ${ }^{14}$ In the present study, the inhibitory effects of chrysin on EAU development were shown for the first time, and we uncovered some of the possible mechanisms that may mediate chrysin's anti-inflammatory effects.

As expected, the chrysin-treated mice exhibited a milder disease phenotype and a significant decrease in their clinical scores compared with those of the control mice, which indicated that chrysin ameliorated the clinical manifestations of EAU in mice. Histopathologically, chrysin inhibited vitreous and retinal infiltration, thus protecting the normal structure of the retina. Our data suggest that chrysin exerts beneficial effects in the treatment of EAU.

Infiltration of inflammatory cells has been reported to be one of the characteristic pathological manifestations of EAU. When self-antigens are released into the body, macrophages or dendritic cells (DCs) recognize and present them to naive $\mathrm{T}$ cells, which are further activated and transformed into $\mathrm{CD} 4^{+}$ Th0 cells. ${ }^{31}$ These $\mathrm{CD} 4^{+}$Th0 cells may differentiate into various other T-cell subsets such as Th1, Th17 and Treg cells, which have many vital roles in a number of autoimmune diseases, including EAU. Early research has indicated that the Th1 cells are the crucial T-cell subset in the onset of EAU. ${ }^{32}$ However, recent studies have indicated that Th17 cells also 

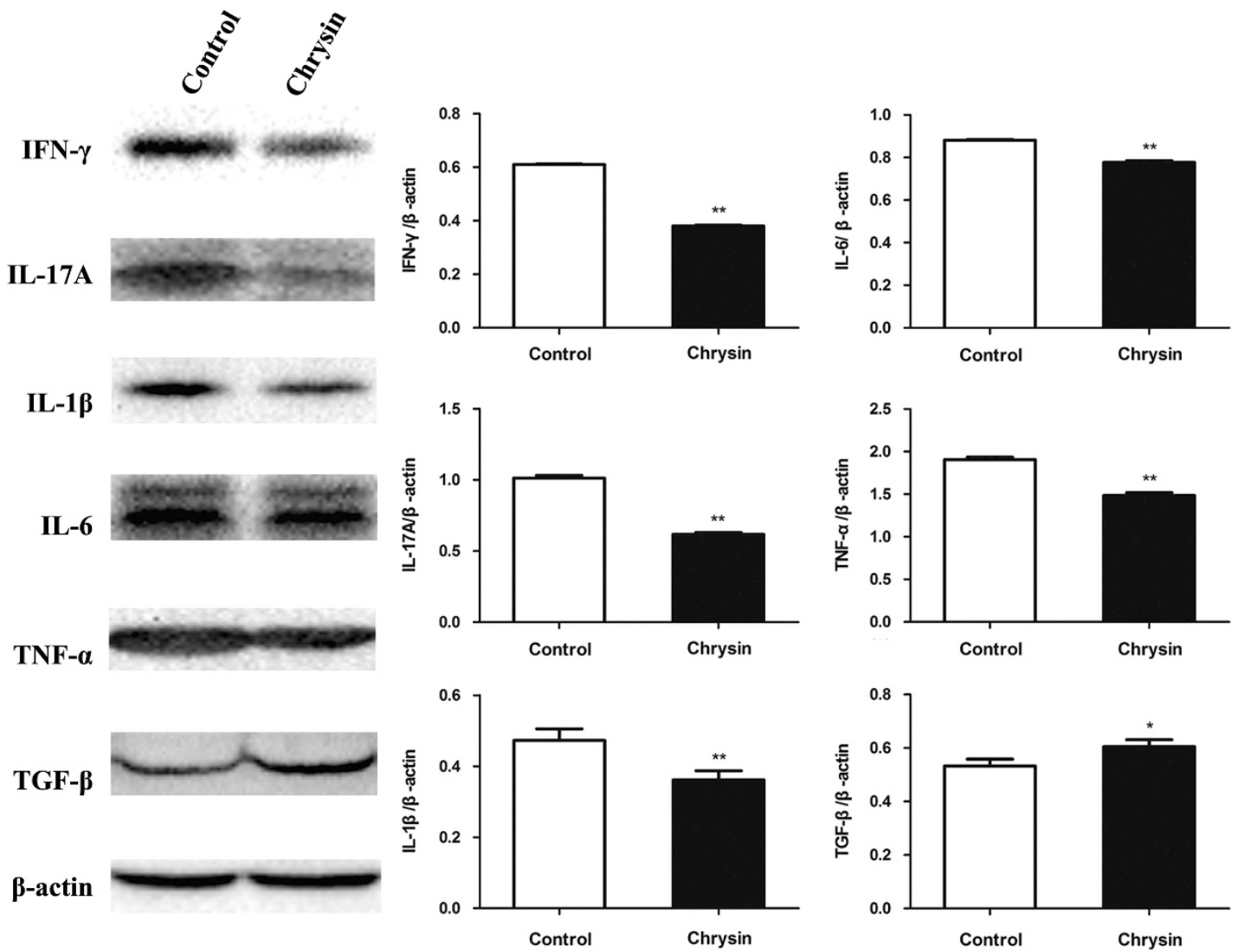

Figure 6 Western blotting measurement of cytokines. Chrysin significantly downregulated IFN- $\gamma$, IL-17A, IL-6, TNF- $\alpha$ and IL-1 $\beta$ but upregulated TGF- $\beta\left({ }^{*} P<0.05 ;{ }^{*} P<0.01 ; n=6\right.$ mice). Data are expressed as the mean \pm s.d. IFN- $\gamma$, interferon- $\gamma$; IL, interleukin; TGF- $\beta$, transforming growth factor- $\beta$; TNF- $\alpha$, tumor necrosis factor- $\alpha$.

\section{Control Chrysin}

p65

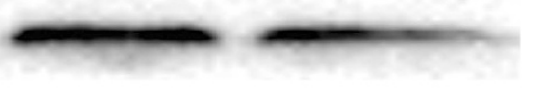

$\beta$-actin
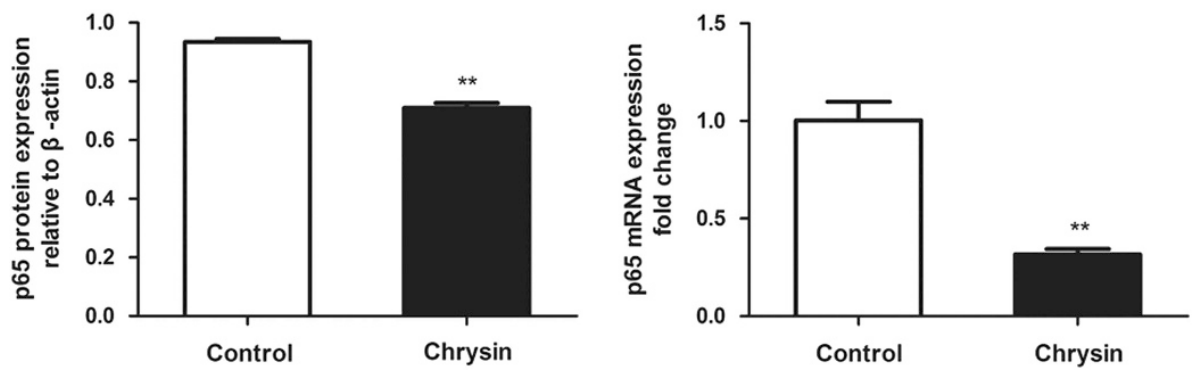

Figure 7 The expression of p65 as shown by western blotting and qRT-PCR. The relative protein and mRNA expression of p65 was inhibited by chrysin (** $P<0.01 ; n=6$ mice). Data are expressed as the mean \pm s.d. qRT-PCR, real-time quantitative PCR.

have the ability to independently induce EAU. ${ }^{33}$ Th1 cells secrete IFN- $\gamma$, whereas Th17 cells mainly secrete IL-17A and IL-1 $\beta$, and Treg cells secrete TGF- $\beta$ and IL-10. Among the multiple cytokines mentioned above, IFN- $\gamma$, IL-17A and IL-1 $\beta$ can help activate and recruit macrophages. The literature contains many reports showing that IL- $1 \beta$ enhances the biased differentiation of $\mathrm{CD} 4^{+} \mathrm{Th} 0$ cells toward Th1 and Th17 cells. ${ }^{34}$ However, TGF- $\beta$ and IL-10 are the main anti-inflammatory 
cytokines responsible for maintaining the homeostasis of the immune system and preventing the occurrence of EAU. In general, Th1 and Th17 cells are considered to be important factors for promoting the pathogenesis of EAU, whereas Treg cells are thought to have a protective effect. These $\mathrm{T}$ cells proliferate in peripheral immune organs, such as the spleen, migrate to the retina, break up the $\mathrm{BRB}$, release inflammatory cytokines and recruit macrophages, thus leading to tissue damage.

Here we used flow cytometry to show that chrysin led to a reduction in the populations of both $\mathrm{CD} 4^{+} \mathrm{IFN}-\gamma^{+}$Th1 cells and $\mathrm{CD}^{+} \mathrm{IL}_{17} 7^{+}$Th17 cells, and induced an increase in $\mathrm{CD} 4^{+}$ CD $25^{+}$Foxp $^{+}$Treg cells in the spleen, which illustrated that chrysin regulates the differentiation of $\mathrm{CD}^{+}$Th0 cells and counterpoises the imbalance in the $\mathrm{T}$ helper-cell subsets. Furthermore, our data showed that the proportion of $\mathrm{CD}^{+}$ Th0 cells decreased after chrysin treatment. This result prompted us to hypothesize that chrysin may inhibit the transformation of naive $\mathrm{T}$ cells into $\mathrm{CD} 4^{+} \mathrm{Th} 0$ cells. Flavonoids are known to exert their immunoregulatory effects by inhibiting the differentiation and function of DCs, ${ }^{35}$ which encouraged us to speculate that chrysin may decrease $\mathrm{CD} 4^{+} \mathrm{Th} 0$ cells by inhibiting the proliferation and function of APCs, such as DCs. However, this hypothesis needs further experimental verification.

In addition, we showed the protective effect of chrysin on the BRB. The BRB, in which occludin and ZO-1 function as important tight junction proteins, is crucial for maintaining the immune privileged status of the eye. ${ }^{36-38}$ The low expression of tight junction proteins, the increased permeability of vessels and the breakdown of the BRB may be the key factors promoting leukocyte recruitment during retinal inflammatory diseases such as EAU. Our current study demonstrated that chrysin maintains the impermeability of the vasculature and protect the integrity of the BRB. The decreased leakage of Evans Blue and higher expression of the tight junction proteins ZO-1 and occludin after chrysin treatment further supported this view.

Macrophages are the main effector cells for tissue damage in EAU. ${ }^{39}$ When the BRB is destroyed, macrophages are recruited and attack intraocular tissues. Two subpopulations of macrophages have been described: M1 macrophages (which exert a destructive effect by producing effector molecules such as iNOS, TNF- $\alpha$, IL- 6 and IL-1 $\beta$ ); and M2 macrophages (which are anti-inflammatory and induce the production of IL-10 and TGF- $\beta$ ). ${ }^{40}$ Activated M1 macrophages promote inflammation and cause tissue damage by enhancing the production of multiple pro-inflammatory cytokines, as described above, and by promoting the release of reactive oxygen species (ROS). Increased expression of iNOS may lead to an imbalance between ROS and antioxidants, causing oxidative stress and tissue damage. Consequently, our group examined the activity of macrophages and found that chrysin inhibited macrophage infiltration in the vitreous and in the retina. Immunofluorescence staining indicated that the activated macrophages that secrete iNOS were suppressed by chrysin treatment. Western blotting and qRT-PCR showed that chrysin downregulated the expression of iNOS, which also supported our hypothesis that chrysin inhibited the activity of macrophages.

The pro-inflammatory cytokines IFN- $\gamma$, IL-17A, IL-6, IL-1 $\beta$ and TNF- $\alpha$ contribute to the recruitment, activation and activity of M1 macrophages, leading to ocular inflammation, whereas TGF- $\beta$ and IL-10 act as anti-inflammatory cytokines. Thus, we evaluated the expression of these cytokines at both the mRNA and protein levels. We observed that chrysin suppressed the levels of IFN- $\gamma$, IL-17A, IL-6, IL-1 $\beta$ and TNF- $\alpha$, but elevated the expression of TGF- $\beta$. Thus, chrysin suppresses intraocular inflammation.

$\mathrm{NF}-\kappa \mathrm{B}$ is a vital nuclear transcription factor for inflammation and regulates the transcription of several inflammatory mediators. It has five subunits: p50, p52, p65, Rel and Rel-B. In the inactive state, these subunits dimerize and bind $\mathrm{I} \kappa \mathrm{B} \alpha$. When cells receive stimuli, such as TNF or IL-1 $\beta$, the dimers (p65-p50 or c-Rel-p50 heterodimers) are released from $\mathrm{I} \kappa \mathrm{B} \alpha$

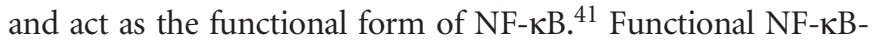
activated macrophages secreting TNF- $\alpha$, iNOS and IL- $1 \beta$ can induce tissue damage. We observed in our study that chrysin suppressed the expression of p65. Therefore, we hypothesize that the translocation of activated NF- $\mathrm{BBp} 65$ to the nucleus may be downregulated in this situation, thus restricting the expression of TNF- $\alpha$, iNOS and IL- $1 \beta$. Our results suggest that chrysin may exert its anti-inflammatory effects on EAU by inhibiting the canonical NF- $\mathrm{KB}$ signaling pathway.

This study revealed a preventive effect of chrysin on EAU and the potential mechanisms underlying this effect. In future studies, we will focus on chrysin's effect on the activation of APCs and their proliferation. We will also study chrysin's effect on upstream stages of T-cell differentiation.

\section{CONCLUSION}

Many studies have presented encouraging evidence for the use of chrysin as a therapeutic intervention in inflammatory diseases. ${ }^{13-17}$ Here, for the first time, we provide evidence that chrysin is an effective oral drug for the treatment of EAU. As an anti-inflammatory molecule, it regulates the imbalance among T-lymphocyte subsets, maintains the integrity of the $\mathrm{BRB}$ and suppresses ocular inflammation, possibly by inhibiting the NF- $\mathrm{KB}$ signaling pathway.

\section{CONFLICT OF INTEREST}

The authors declare no conflict of interest.

\section{ACKNOWLEDGEMENTS}

This study was supported by the National Natural Science Foundation of China (grant numbers 81371038 and 91442124), the Natural Science Foundation of Tianjin (grant 12JCYBJC33900 and 14JCYBJC28000), and TMUGH (grant number ZYYFY2015026). We thank Tim Corson and the Volunteer Editor Program for providing expert assistance with English language. The protocol for the research project has been approved by the ethics committee of the hospital, and the research conforms to the provisions of the ARVO statement for animal research. 
1 Chang JH, Wakefield D. Uveitis: a global perspective. Ocul Immunol Inflamm 2002; 10: 263-279.

2 Selmi C. Diagnosis and classification of autoimmune uveitis. Autoimmun Rev 2014; 13: 591-594.

3 Davatchi F, Sadeghi Abdollahi B, Shams H, Shahram F, Nadji A, Chams-Davatchi $\mathrm{C}$ et al. Combination of pulse cyclophosphamide and azathioprine in ocular manifestations of Behcet's disease: longitudinal study of up to 10 years. Int J Rheum Dis 2014; 17: 444-452.

4 Cervantes-Castaneda RA, Gonzalez-Gonzalez LA, Cordero-Coma M, Yilmaz T, Foster CS. Combined therapy of cyclosporine A and mycophenolate mofetil for the treatment of birdshot retinochoroidopathy: a 12-month follow-up. Br J Ophthalmol 2013; 97: 637-643.

5 Al-Rayes H, Al-Swailem R, Al-Balawi M, Al-Dohayan N, Al-Zaidi S, Tariq M. Safety and efficacy of infliximab therapy in active behcet's uveitis: an open-label trial. Rheumatol Int 2008; 29: 53-57.

6 Sobrin L, Kim EC, Christen W, Papadaki T, Letko E, Foster CS. Infliximab therapy for the treatment of refractory ocular inflammatory disease. Arch Ophthalmol 2007; 125: 895-900.

7 Haruta H, Ohguro N, Fujimoto M, Hohki S, Terabe F, Serada S et al. Blockade of interleukin- 6 signaling suppresses not only th17 but also interphotoreceptor retinoid binding protein-specific Th1 by promoting regulatory $\mathrm{T}$ cells in experimental autoimmune uveoretinitis. Invest Ophthalmol Vis Sci 2011; 52: 3264-3271.

8 Luger D, Silver PB, Tang J, Cua D, Chen Z, Iwakura Y et al. Either a Th17 or a Th1 effector response can drive autoimmunity: conditions of disease induction affect dominant effector category. J Exp Med 2008; 205: 799-810.

9 Wang $L, X u Y, Y u$ Q, Sun Q, Xu Y, Gu Q et al. H-RN, a novel antiangiogenic peptide derived from hepatocyte growth factor inhibits inflammation in vitro and in vivo through $\mathrm{PI} 3 \mathrm{~K} / \mathrm{AKT} / \mathrm{IKK} / \mathrm{NF}-\mathrm{kappaB}$ signal pathway. Biochem Pharmacol 2014; 89: 255-265.

10 Yang X, Jin H, Liu K, Gu Q, Xu X. A novel peptide derived from human pancreatitis-associated protein inhibits inflammation in vivo and in vitro and blocks NF-kappa B signaling pathway. PLoS One 2011; 6: e29155.

11 Lv PC, Cai TT, Qian Y, Sun J, Zhu HL. Synthesis, biological evaluation of chrysin derivatives as potential immunosuppressive agents. Eur J Med Chem 2011; 46: 393-398.

12 Middleton Jr E, Kandaswami C, Theoharides TC. The effects of plant flavonoids on mammalian cells: implications for inflammation, heart disease, and cancer. Pharmacol Rev 2000; 52: 673-751.

13 Nagaki Y, Hayasaka S, Kadoi C, Nakamura N, Hayasaka Y. Effects of scutellariae radix extract and its components (baicalein, baicalin, and wogonin) on the experimental elevation of aqueous flare in pigmented rabbits. Jpn J Ophthalmol 2001; 45: 216-220.

14 Shiratori K, Ohgami K, Ilieva I, Jin XH, Yoshida K, Kase S et al. The effects of naringin and naringenin on endotoxin-induced uveitis in rats. J Ocul Pharmacol Ther 2005; 21: 298-304.

15 Shin EK, Kwon HS, Kim YH, Shin HK, Kim JK. Chrysin, a natural flavone, improves murine inflammatory bowel diseases. Biochem Biophys Res Commun 2009; 381: 502-507.

16 Bae Y, Lee S, Kim SH. Chrysin suppresses mast cell-mediated allergic inflammation: involvement of calcium, caspase-1 and nuclear factorkappaB. Toxicol App/ Pharmacol 2011; 254: 56-64.

17 Du Q, Gu X, Cai J, Huang M, Su M. Chrysin attenuates allergic airway inflammation by modulating the transcription factors T-bet and GATA-3 in mice. Mol Med Rep 2012; 6: 100-104.

18 Dou W, Zhang J, Zhang E, Sun A, Ding L, Chou G et al. Chrysin ameliorates chemically induced colitis in the mouse through modulation of a PXR/NF-kappaB signaling pathway. J Pharmacol Exp Ther 2013; 345: 473-482.

19 Xiao J, Zhai H, Yao Y, Wang C, Jiang W, Zhang C et al. Chrysin attenuates experimental autoimmune neuritis by suppressing immunoinflammatory responses. Neuroscience 2014; 262: 156-164.
20 Agarwal RK, Silver PB, Caspi RR. Rodent models of experimental autoimmune uveitis. Methods Mol Biol 2012; 900: 443-469.

21 Livak KJ, Schmittgen TD. Analysis of relative gene expression data using real-time quantitative PCR and the 2(-Delta Delta C(T)) Method. Methods 2001; 25: 402-408.

22 Bettelli E, Carrier Y, Gao W, Korn T, Strom TB, Oukka M et al. Reciprocal developmental pathways for the generation of pathogenic effector TH17 and regulatory T cells. Nature 2006; 441: 235-238.

23 Ferraro A, Socci C, Stabilini A, Valle A, Monti P, Piemonti L et al. Expansion of Th17 cells and functional defects in T regulatory cells are key features of the pancreatic lymph nodes in patients with type 1 diabetes. Diabetes 2011; 60: 2903-2913.

24 Korn T, Mitsdoerffer M, Croxford AL, Awasthi A, Dardalhon VA, Galileos $\mathrm{G}$ et al. IL-6 controls Th17 immunity in vivo by inhibiting the conversion of conventional T cells into Foxp3+ regulatory T cells. Proc Natl Acad Sci USA 2008; 105: 18460-18465.

25 Niu Q, Cai B, Huang ZC, Shi YY, Wang LL. Disturbed Th17/Treg balance in patients with rheumatoid arthritis. Rheumatol Int 2012; 32 . 2731-2736.

26 Szodoray P, Nakken B, Barath S, Csipo I, Nagy G, El-Hage F et al. Altered Th17 cells and Th17/regulatory T-cell ratios indicate the subsequent conversion from undifferentiated connective tissue disease to definitive systemic autoimmune disorders. Hum Immunol 2013; 74: $1510-1518$.

27 Zhou L, Lopes JE, Chong MM, Ivanov II, Min R, Victora GD et al. TGF-beta-induced Foxp3 inhibits $\mathrm{T}(\mathrm{H}) 17$ cell differentiation by antagonizing RORgammat function. Nature 2008; 453: 236-240.

28 Rahimi M, Moinfar N, Ashrafi A. The potential benefits of green tea in patients with uveitis. Med Hypotheses 2007; 69: 702-703.

29 Qin YJ, Chu KO, Yip YW, Li WY, Yang YP, Chan KP et al. Green tea extract treatment alleviates ocular inflammation in a rat model of endotoxin-induced uveitis. PLoS One 2014; 9: e103995.

30 Pescosolido N, Giannotti R, Plateroti AM, Pascarella A, Nebbioso M. Curcumin: therapeutical potential in ophthalmology. Planta Medica 2014; 80: 249-254.

31 Liberal R, Longhi MS, Mieli-Vergani G, Vergani D. Pathogenesis of autoimmune hepatitis. Best Pract Res Clin Gastroenterol 2011; 25: 653-664.

32 Caspi RR. Th1 and Th2 responses in pathogenesis and regulation of experimental autoimmune uveoretinitis. Int Rev Immunol 2002; 21: 197-208.

33 Amadi-Obi A, Yu CR, Liu X, Mahdi RM, Clarke GL, Nussenblatt RB et al. TH17 cells contribute to uveitis and scleritis and are expanded by IL-2 and inhibited by IL-27/STAT1. Nat Med 2007; 13: 711-718.

34 Zhao R, Zhou H, Zhang J, Liu X, Su SB. Interleukin-1beta promotes the induction of retinal autoimmune disease. Int Immunopharmacol 2014; 22: 285-292.

35 Nickel T, Hanssen H, Sisic Z, Pfeiler S, Summo C, Schmauss D et al. Immunoregulatory effects of the flavonol quercetin in vitro and in vivo. Eur J Nutr 2011; 50: 163-172.

36 Nicholson D, Kerr EC, Jepps OG, Nicholson LB. Modelling experimental uveitis: barrier effects in autoimmune disease. Inflamm Res 2012; 61: 759-773.

37 Xu H, Dawson R, Crane IJ, Liversidge J. Leukocyte diapedesis in vivo induces transient loss of tight junction protein at the blood-retina barrier. Invest Ophthalmol Vis Sci 2005; 46: 2487-2494.

38 Mochizuki M. Intraocular inflammation and homeostasis of the eye. Nihon Ganka Gakkai Zasshi 2009; 113: 344-377. Discussion 378.

39 Dagkalis A, Wallace C, Xu H, Liebau S, Manivannan A, Stone MA et al. Development of experimental autoimmune uveitis: efficient recruitment of monocytes is independent of CCR2. Invest Ophthalmol Vis Sci 2009; 50: 4288-4294.

40 Gordon S. Alternative activation of macrophages. Nat Rev Immunol 2003; 3: 23-35.

41 Hayden MS, Ghosh S. NF-kappaB, the first quarter-century: remarkable progress and outstanding questions. Genes Dev 2012; 26: 203-234. 